http://www.jfas.info

\title{
PERFORMANCE OF MODIFIED POWER SPECTRAL DENSITY FEATURES IN EEG SIGNAL CLASSIFICATION
}

\author{
F. H. K. Zaman ${ }^{1, *}$, N. A. M. Shukur ${ }^{1}$, N. $\operatorname{Hamzah}^{1}$, N. M. Zaini ${ }^{1}$ and Z. I. $\operatorname{Rizman}^{2}$ \\ ${ }^{1}$ Faculty of Electrical Engineering, UniversitiTeknologi MARA, 40450 Shah Alam, Selangor, \\ Malaysia \\ ${ }^{2}$ Faculty of Electrical Engineering, UniversitiTeknologi MARA, 23000 Dungun, Terengganu, \\ Malaysia
}

Published online: 10 September 2017

\begin{abstract}
This paper evaluates the performance of classification of Electroencephalogram (EEG) data by focusing on several normalization and dimensionality reduction processes in Power Spectral Density (PSD) signal pre-processing. It focuses on effect of modification of PSD features as an input for classification of EEG signals. For ANN classification, Zero-mean normalization method produces the best performance when compared against other complicated dimensionality reduction techniques such as Locally Linear Embedding (LLE) and Orthogonal Least Squares (OLS). The improvement achieved by Zero-mean normalization in ANN is $4.5 \%$ better than Baseline PSD. For SVM classification, PCA produces best performance with an enhancement as much as $10 \%$ better than Baseline PSD. It found that SVM classifier performs significantly better than ANN classifier in classifying variants of PSD features.
\end{abstract}

Keywords: feature extraction; dimensionality reduction; PSD; EEG.

\footnotetext{
Author Correspondence, e-mail: fadhlan@salam.uitm.edu.my doi: http://dx.doi.org/10.4314/jfas.v9i3s.65
} 


\section{INTRODUCTION}

The brain of a human is usually referred as a wonderful and the most powerful processor in the world. To protect the precious processor, the skin and the skull in the head are the one who responsible for the role. Nonetheless, these organs are recognized as one of the excellent electrical insulators and making the recording of data from a single neuron in the brain is almost impossible to be done without any special device. To develop this device, a lot of research has been done. In 1930s, Hans Berger, the first human who discovered a new method named 'Electroencephalography' (EEG) [1] to collect the signal from the neurons in the brain and he is the first human subject being tested using EEG.

EEG is signals that provide ample information regarding the electrical activity of human brain. The method requires the process of capturing or recording the electrical signals from the neurons in the brain needs to be executed from scalp, where the frequency and the amplitudes will be varies according to the current task that being done by the brain. Thereby, another application can be used to recognize and classify the signals is the Brain Computer Interface (BCI). BCI is an artificial intelligence system that combine both hardware and software of the communication system and capable to recognize a certain set of patterns in the signals of the brain comprising of five stages which are the signal acquisition, preprocessing or signal enhancement, feature extraction, classification and the control interface [2, 22]. Table 1 states the roles on the five stages.

BCI device can read mental commands represented by EEG signals and transforming them into real commands which can be used in many autonomous applications. Some of the applications of BCI are wheelchair control [3-4], unmanned aerial vehicle [5-6], home appliances [7], robot [8] and vehicle control approaches [9-10]. One of the most important components of $\mathrm{BCI}$ is the feature extraction of EEG signals. The performance of classification of mental tasks relies heavily on this procedure, where methods such as Power Spectral Density (PSD) and Power Spectral Entropy (PSE) [10] are used.

This paper aims to identify the classification performance of PSD between two type of features; raw PSD and modified PSD features which can be achieved through several dimensionality reduction and normalization techniques that uses a compact feature vector re-mapping that represents the unique or interesting specifications of an image or data [11]. We propose to use Artificial Neural Network (ANN) and Support Vector Machines (SVM) as the classifier. However, since the process of the EEG signal acquisition are challenging, 
several studies are required prior classification.

Table 1. Roles of the five stages of signal recognition

\begin{tabular}{cl}
\hline Stages & \multicolumn{1}{c}{ Function } \\
\hline Signal Acquisition & Capturing the brain signals and performing noise reduction (filtering) \\
Pre-processing & Preparing the signal into a suitable form to be used for further \\
(Signal & processing. \\
Enhancement) &
\end{tabular}

Feature Extraction Identifies, measuring and plotting the brain signals into several discriminant features from the signals.

Classification Classifies the features and choosing the 'good' features to achieve effective pattern recognition.

Control Interface Translate the signals that have been classified into meaningful and useful commands to be used for the developments of new devices.

PSD is one of the powerful methods to identify the repetitive and correlated signals [12-13]. Generally, PSD describes how the power of a signal is distributed with frequency. The power spectral density are shows the strength of the variations (energy) as a function of frequency [14]. The computed data using PSD are known as periodogram. Power Spectral Density is mainly used to analyze the location of alpha rhythm. It is observed that when the location of electrode is changed from frontal to parietal lobes, the peak of alpha rhythm is decrease. The person with closed eyes have more dominant wave of alpha rhythm over the back of the head [11].

Fig. 1 shows the Emotiv EPOC with 16 sensors, one which is being used in our experiment for the data acquisition process. The shape of this device is similar with headphones and there are 16 independent sensors attached to the Emotiv EPOC (only 14 are used in the experiment). These sensors consist of felt pads with gold connections to increase the sensitivity of the pickups. One of the challenges of using this device is that the felt sensors need to be moist at all time during the process of data acquisition to conduct the potential difference across the skull and the solution that can be used is the saline solution [15-16].

The signals were recorded and executed to analyze the accuracy of classification of two actions-lifting left and right hand-based on many variants PSD features. To acquire the raw PSD features, the data will undergo the feature extraction process which is the Power Density 
Spectrum. Table 2 explains briefly on the different bands of EEG signal together with their frequency ranges and activities description. Next, the extracted data will then be transferred into ANN and SVM classifier to obtain the classification accuracy of PSD features.
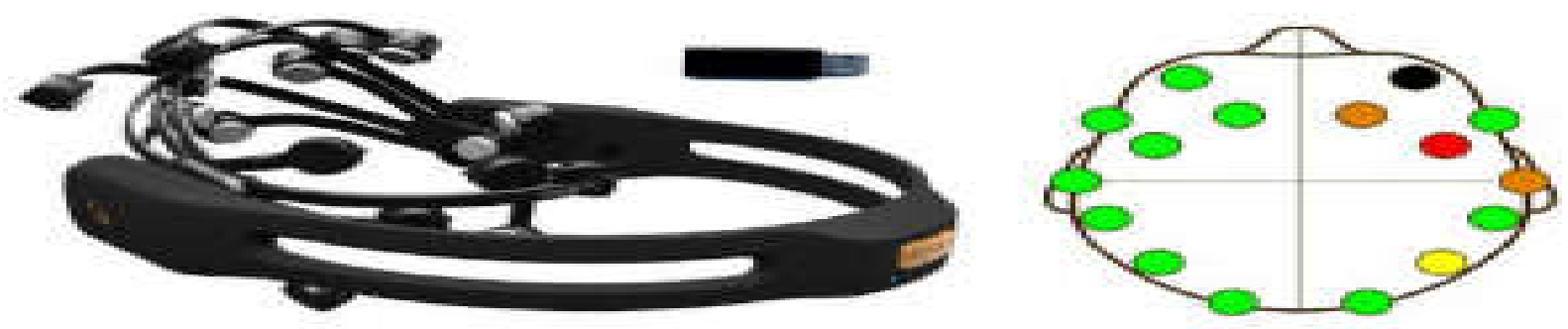

Fig.1.Emotiv EPOC neuroheadset and the locations of its 16 sensor [16]

Table 2. Recognition brain wave pattern

\begin{tabular}{ccc}
\hline Band & Frequency Range $(\mathbf{H z})$ & Activity of the Subject \\
\hline Delta & $0.5-3$ & Sleep \\
Theta & $4-7$ & Mental imagery \\
Alpha & $8-12$ & Relaxation, Sensory and Motor Activity \\
Beta & $12-30$ & Active Concentration and Motor Idling \\
Gamma & $26-100$ & Cognitive and Motor Functions \\
\hline
\end{tabular}

\section{METHODOLOGY}

\subsection{Overall Working Principle for EEG Signal}

This section presents on the sequence procedures adopted in this paper. Fig. 2 shows the flowchart for overall process that being carried out in this paper. The further detail regarding different stage in this flowchart was elaborated in the section below.

\subsection{Experimental Procedure to Acquire Raw EEG Data}

In this work, the experiment is conducted in which a person is asked to lift their left or right hand for 30 seconds with eyes opened. Further explanation of the experiment is shown in Table 3.

\subsection{Filtering}

The feature extraction process begins with the implementation of band pass filtering in order to remove any unwanted noise and artefacts. Artefacts such as eye-blinking, electrocardiograms (ECGs), breathing from respiratory system and any other internal or external noise will affect the result analysis. Hence, we use band pass filter to filter out the 
unwanted noise and signal. In this experiment, raw data has been filtered from 25 to 35 seconds to have accurate value for EEG signal when the changes take place.

\subsection{Feature Extraction}

The performance of the classification is dependent on the feature extraction since the extracted features from EEG signal will help classifier to classify the different mental tasks based on the different patterns captured in the EEG signal. In this work, PSD is used as feature extraction method. PSD values are computed by using the mean data ratio of each band. The dimensionality reduction and normalization of PSD data is carried out using magnitude scaling (mag-scale) (1), softmax scaling (2), Zero-mean normalization (3), outliers removal by Orthogonal Least Squares (OLS) [17], dimensionality reduction using Locally Linear Embedding (LLE) [18] and Principal Component Analysis (PCA):

$$
\begin{aligned}
& \mathrm{PSD}_{\text {mag-scale }}=\frac{\mathrm{PSD}-\mathrm{PSD}_{\min }}{\mathrm{PSD}_{\max }-\mathrm{PSD}_{\min }} \\
& \mathrm{PSD}_{\text {softmax }}=\frac{\log (\mathrm{PSD}+1)}{\log \left(\mathrm{PSD}_{\min }+1\right)} \\
& \mathrm{PSD}_{\text {zero-mean }}=\left(\frac{1}{\mathrm{PSD}_{\text {std }}}\right)\left(\mathrm{PSD}-\mathrm{PSD}_{\text {mean }}\right)
\end{aligned}
$$

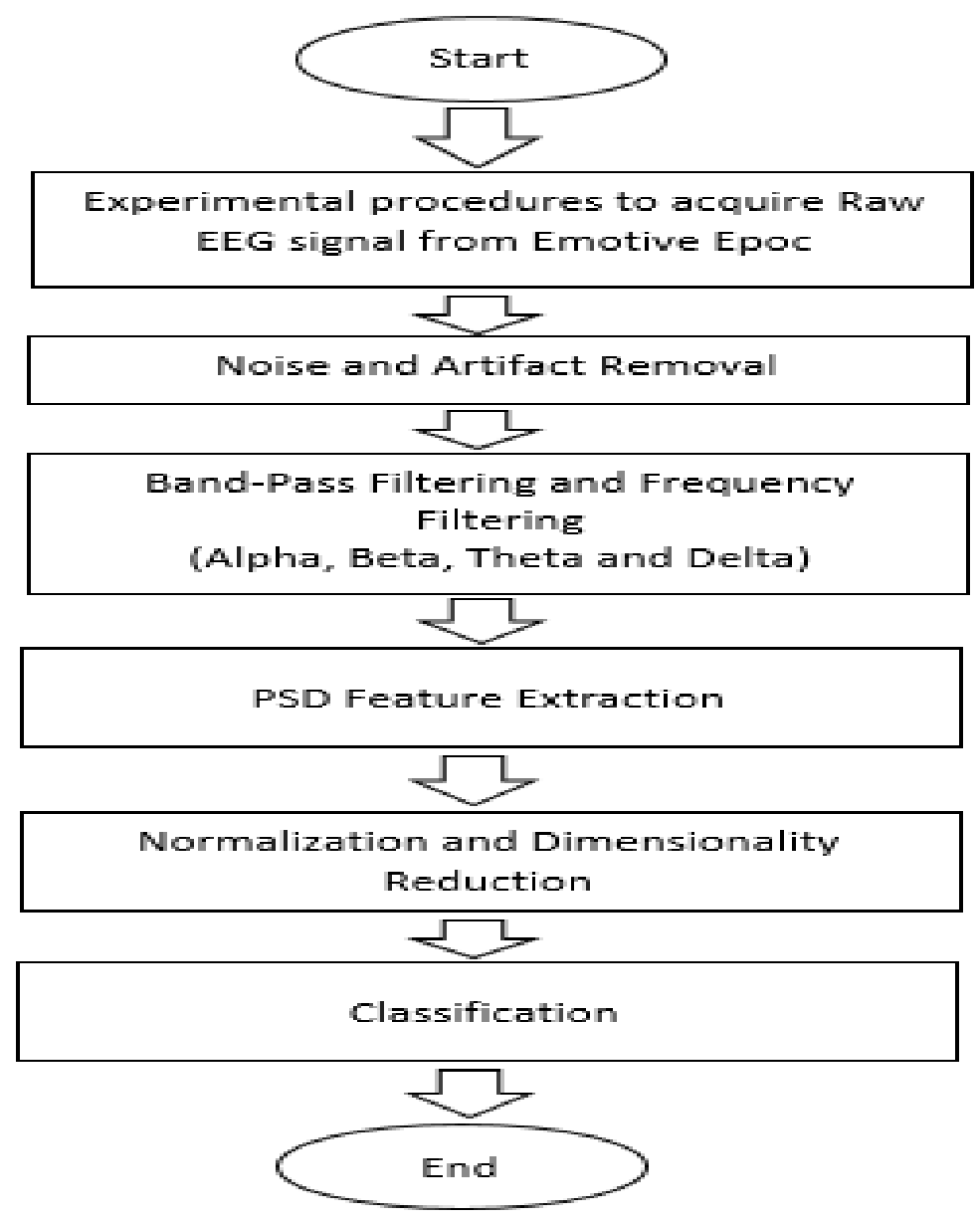

Fig.2. Flowchart of proposed method 


\subsection{Classification Using ANN and SVM}

In this paper, ANN is constructed and later evaluated for accuracy, specificity and sensitivity in classifying the given EEG signal data in the form of PSD feature values. MATLAB and Simulink Toolbox will be used especially for signal processing purpose. For SVM, LibSVM toolbox [19] is used to simplify the implementation in MATLAB. The number of input features are determined based on the number of input nodes, while the number of different target function (1 output: left or right) determine the number of output nodes.

Table 3. Experimental procedure

\begin{tabular}{|c|c|}
\hline Right & - Person relaxes their mind for 1 minute \\
\hline Hand & $\begin{array}{l}\text { - Person lifts their right hand once for every } 30 \text { second. The data is being } \\
\text { recorded and save. This process is being repeated for } 20 \text { times with open eyes }\end{array}$ \\
\hline & - Person relaxes their mind for 1 minute \\
\hline
\end{tabular}

Left Hand - Person relaxes their mind for 1 minute.

- Person lifts their left hand once for every 30 second. The data is being recorded and save. This process is being repeated for 20 times with open eyes.

- Person relaxes their mind for 1 minute.

ANN can be classified either as Single-layer Perceptron or Multi-Layer Perceptron Neural Network (MLPNN). SLP is known to have many limitations and in this regards some researchers have found that combining the single perceptron might be useful for a larger network. Thus, for our study, MLPNN is believed to provide a better solution. The number of inputs denotes the number of features selected, while the number of outputs denotes the number of classes formed.

We can see that there is for input signal which is alpha, beta, theta and delta frequency band. The number of neurons in hidden layer use is set between range 5 to 100 for all the data set to determine, which number of hidden layer neurons that will produce the highest accuracy. The number of output neuron is 2 since we have two different classes which is left and right.

\subsection{Performance Evaluation}

After the EEG signal has been classified, we need to evaluate the accuracy of the PSD feature classification for both ANN and SVM classifier. The result obtained is in term of accuracy inside the confusion matrix as shown in Fig. 3. 


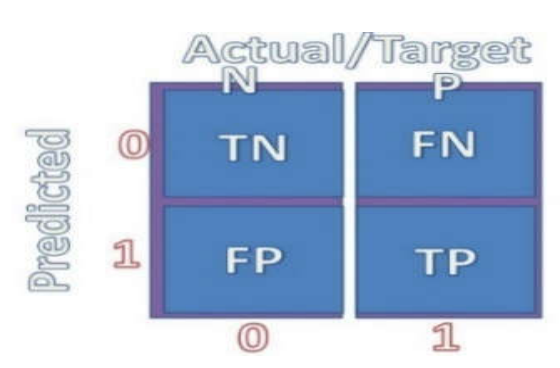

Fig.3. Confusion matrix

We use confusion matrix to validate the data classification performance. There are two output classes which are left or right command. We are producing three set of confusion matrix based on the physical, imaginary and combination of both data. To illustrate, there are 4 terms used inside the confusion matrix which are True Positive (TP), False Negative (FN), False Positive (FP) and True Negative (TN). We set zero as the right hand, whiles the one as the left hand. To elaborate, TP is the section where the network predicted right command and the subject were doing the right command activity while $\mathrm{TN}$ is the section when the network predicted left command and the subject were doing the left command activity. For FP also known as "Type I error" section is when the network predicted right command, but the subject was doing the left command activity while FN also known as “Type II error" section is when the network predicted left command but the subject was doing the right command activity.

\section{RESULTS AND DISCUSSION}

This section will further discuss on the findings that we acquired when conducting the experiment. After the data is being extracted using PSD, the data is processed with several feature extraction and normalization techniques before being fed as an input for MLPNN and SVM Classifier. MLPNN is one of the common classification methods in data mining. MLPNN is a feed forward network that makes a model to map input data to output data. Hidden layer in MLP can include various layers between input and output.

Besides the EEG data acquired from Emotive Epoc BCI device, we use another publicly available dataset provided by IDIAP Research Institute [20] which is available from the link here: http://bbci.de/competition/iii/desc_V.html. This dataset contains data from 3 normal subjects during 4 non-feedback sessions. The subjects sat in a normal chair, relaxed arms resting on their legs. There are 3 tasks namely (1) Imagination of repetitive self-paced left hand movements, (2) Imagination of repetitive self-paced right hand movements, and (3) Generation of words beginning with the same random letter. We use the pre-computed PSD 
signals from subject 1 only in this experiment. We denote the manually acquired EEG data as Dataset 1, while the other dataset as Dataset 2.

In the experiment, the accuracy of the classification is measured in 10 trials for each different number of hidden neuron used as well as for SVM run. The average accuracy from MLPNN and SVM classification for Dataset 1 and Dataset 2 is then recorded in Table 4, Table 5 and Table 6 respectively. The parameters used in this experiment are as follows: for MLPNN, scaled conjugate gradient is used as learning rule. SVM configuration is set such that the nu-SVC type and RBF kernel is used. The datasets are randomly divided such that $50 \%$ of the data is used as training set, $20 \%$ used as validation set and $30 \%$ used as test set. The number of retained OLS features are 30, while the retained features for LLE is 10 and its neighbourhood $\mathrm{K}$ is 10 . For PCA, the number of retained features is 10 .

Table 4. Performance of PSD classification using MLPNN for Dataset 1

\begin{tabular}{cccccccc}
\hline Hidden & & \multicolumn{7}{c}{ Average Accuracy (\%) } \\
Neurons & Baseline & Mag-Scale & Softmax & Zero-Mean & OLS & LLE & PCA \\
& PSD & PSD & PSD & PSD & PSD & PSD & PSD \\
\hline 5 & 43.00 & 52.00 & $\mathbf{6 5 . 0 0}$ & 55.25 & 59.50 & 57.00 & 55.00 \\
10 & 47.50 & 54.75 & 60.75 & 51.75 & 53.75 & 54.50 & 46.75 \\
15 & 56.25 & 50.00 & 59.00 & $\mathbf{6 6 . 0 0}$ & 53.00 & 54.50 & 48.25 \\
20 & 57.00 & 56.75 & 48.25 & 51.75 & 54.00 & 52.25 & 53.00 \\
25 & 51.50 & 53.25 & 42.50 & 52.50 & 52.25 & 58.75 & 48.25 \\
30 & 50.25 & 50.25 & 50.25 & 54.50 & 46.25 & $\mathbf{6 0 . 5 0}$ & 51.50 \\
35 & 50.75 & 46.75 & 54.75 & 49.75 & 47.50 & 51.25 & 48.00 \\
40 & 55.25 & 63.50 & 52.75 & 52.00 & 56.50 & 53.50 & 42.00 \\
45 & $\mathbf{6 1 . 5 0}$ & 55.75 & 47.75 & 49.75 & $\mathbf{6 0 . 5 0}$ & 48.50 & 50.25 \\
50 & 54.50 & 57.75 & 41.00 & 44.75 & 52.75 & 46.50 & 53.50 \\
55 & 49.75 & $\mathbf{6 4 . 5 0}$ & 42.25 & 49.75 & 48.50 & 49.50 & $\mathbf{5 5 . 5 0}$ \\
60 & 59.25 & 61.00 & 53.25 & 55.25 & 51.25 & 52.75 & 53.50 \\
65 & 51.00 & 52.75 & 55.25 & 48.50 & 59.50 & 60.50 & 52.00 \\
70 & 54.25 & 42.25 & 49.50 & 54.75 & 54.50 & 55.75 & 47.50 \\
75 & 53.25 & 53.25 & 47.00 & 47.25 & 46.75 & 55.25 & 54.00
\end{tabular}




\begin{tabular}{cccccccc}
80 & 54.75 & 49.50 & 55.75 & 47.75 & 51.50 & 46.50 & 54.25 \\
85 & 51.00 & 49.75 & 55.75 & 49.00 & 46.50 & 51.25 & 54.25 \\
90 & 59.25 & 47.00 & 52.00 & 48.25 & 53.50 & 46.25 & 47.50 \\
95 & 55.00 & 56.50 & 55.00 & 55.75 & 51.00 & 50.50 & 49.50 \\
100 & 44.75 & 56.25 & 51.50 & 58.75 & 51.50 & 55.50 & 54.25 \\
\hline
\end{tabular}

Table 5. Performance of PSD classification using SVM for Dataset 1

\begin{tabular}{cc}
\hline Method & Average Accuracy (\%) \\
\hline Baseline PSD & 81.67 \\
Mag-Scale PSD & 80.83 \\
Softmax PSD & 70.83 \\
Zero-mean PSD & 87.50 \\
OLS PSD & 80.83 \\
LLE PSD & 82.50 \\
PCA PSD & $\mathbf{9 1 . 6 7}$ \\
\hline
\end{tabular}

Table 6. Performance of PSD classification using SVM for Dataset 2

\begin{tabular}{cc}
\hline Method & Average Accuracy (\%) \\
\hline Baseline PSD & 74.08 \\
Mag-Scale PSD & 74.21 \\
Softmax PSD & 39.54 \\
Zero-mean PSD & $\mathbf{7 5 . 0 7}$ \\
OLS PSD & 39.95 \\
LLE PSD & 60.77 \\
PCA PSD & 73.35
\end{tabular}

From Table 4, it is observed that feature modification process produces better performance compared to the raw PSD features (shown in Table 4 as 'Baseline'). The highest accuracy is achieved by Zero-mean normalization process at $66.00 \%$ accuracy, while other methods such as Softmax normalization and Mag-Scale normalization produces $65.00 \%$ and $64.50 \%$ accuracy respectively. Baseline PSD feature manages to produce $61.50 \%$ accuracy, while more complicated modification procedures such as LLE and OLS produces worse result at $60.50 \%$ accuracy for both. It is observed that Zero-mean normalization produces $4.5 \%$ improvement in term of classification accuracy when compared against Baseline PSD (un-normalized PSD). 
It is also observed that the number of neurons in hidden layer also plays important role is determining the accuracy of MLPNN classification. The best number of neurons in hidden layer for Baseline, Mag-Scale, Softmax and Zero-mean are 45, 55, 5 and 15 respectively. On the other hand, for OLS, LLE and PCA, best performance is acquired when the number of neurons in hidden layers are 30, 45 and 55 respectively.

According to Table 5, the best accuracy of $91.67 \%$ for SVM classifier is acquired when PSD features are reduced using PCA. This is $10 \%$ better than Baseline PSD at $81.67 \%$ accuracy. When compared against MLPNN classification, best performance of MLPNN which is at $66 \%$ accuracy (Zero-mean) is much worse than SVM's 91.67\% accuracy (PCA). Based on Table 6, the best SVM classification accuracy of $75.07 \%$ is achieved by Zero-mean normalization which is just $1 \%$ better than Baseline PSD.

Fig. 4 and 5 show the confusion matrix and the MLPNN performance of the training, validation, test and overall process for with unnormalized and Zero-mean PSD feature respectively. There are 4 confusion matrices obtained when we train the network which is training, validation, test and all. The training process presented to the network and the network is adjusted per its error. Validation process are used for measure network generalization and to halt training when generalization stop improving, while testing process provide an independent measurement of network performance during and after training.
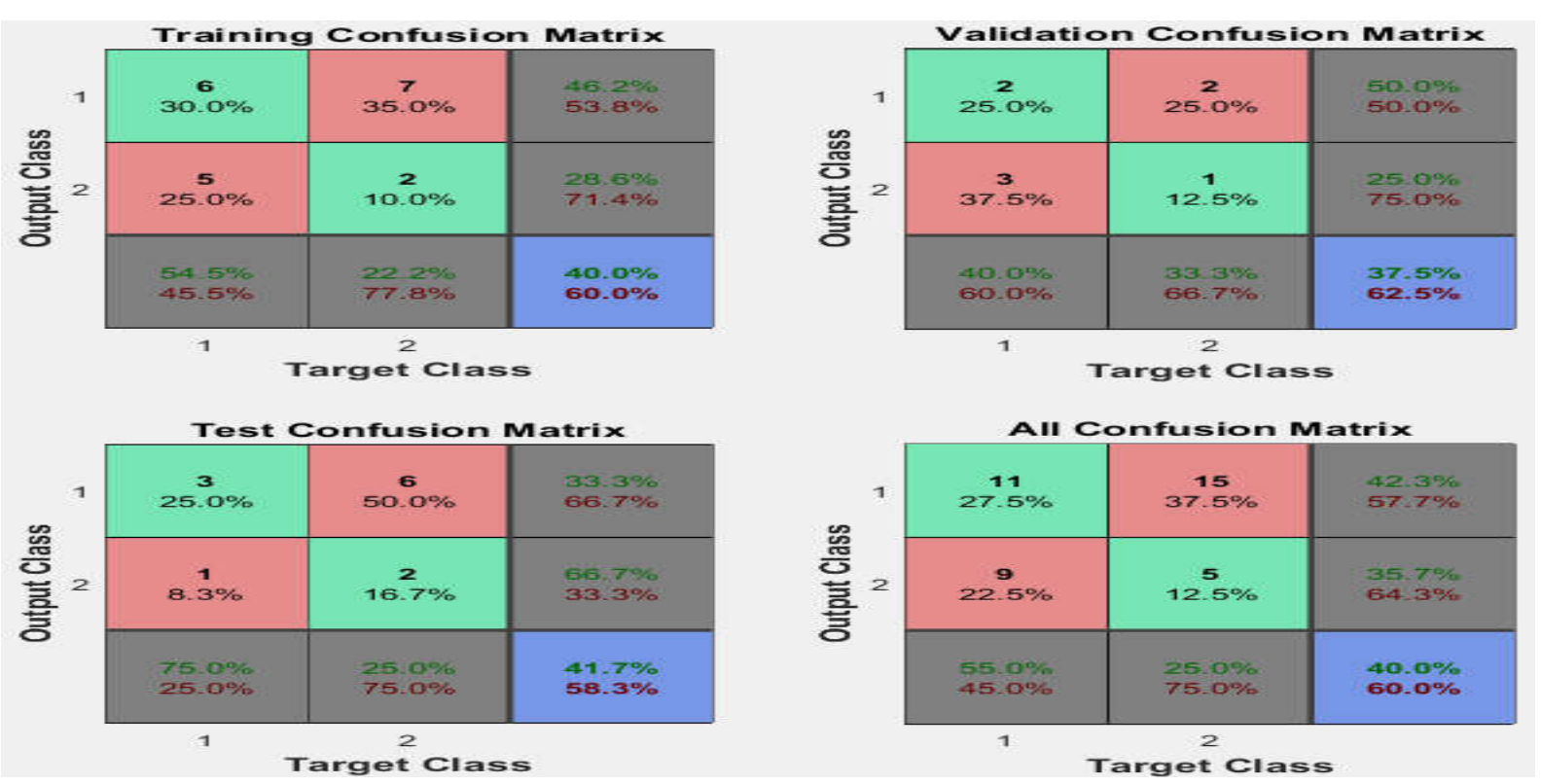

(a) 


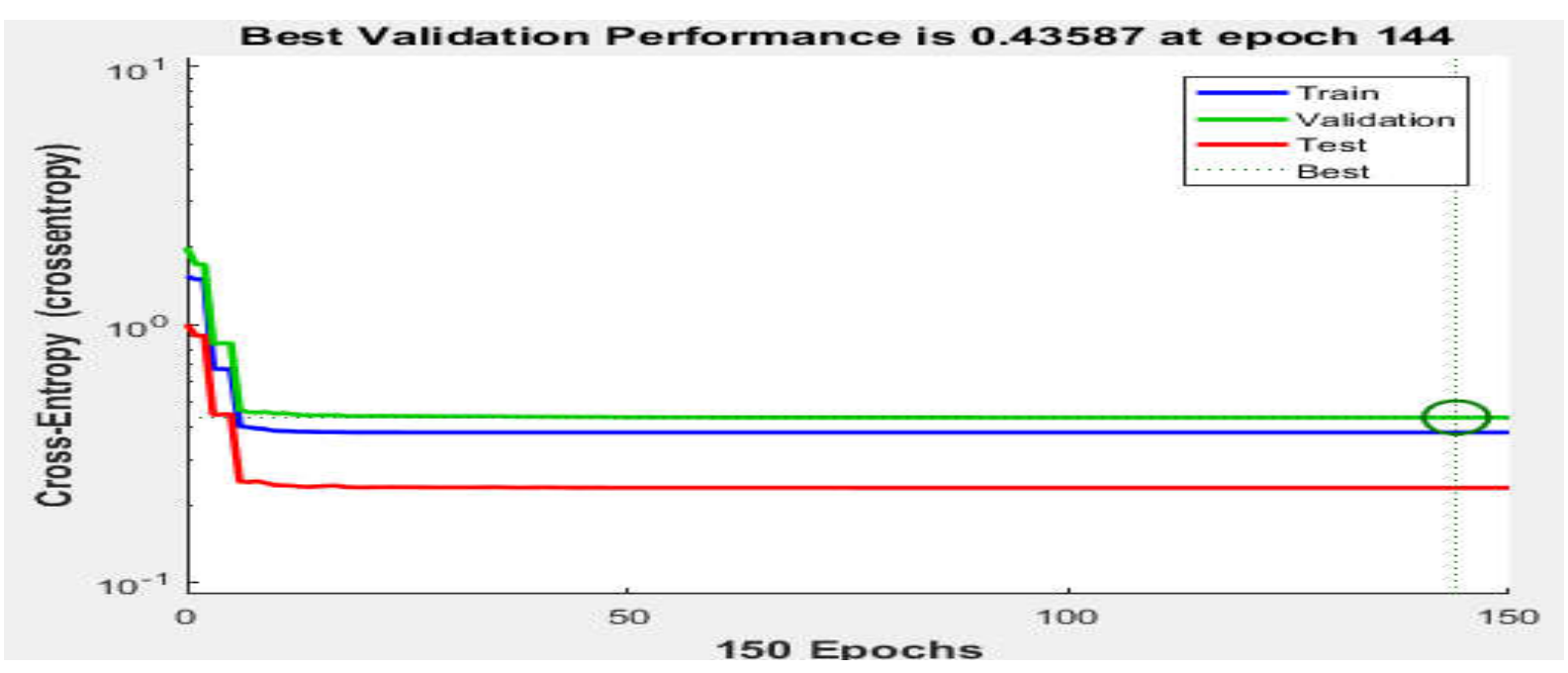

(b)

Fig.4. (a) Confusion Matrix and (b) MLPNN performance for Baseline PSD feature
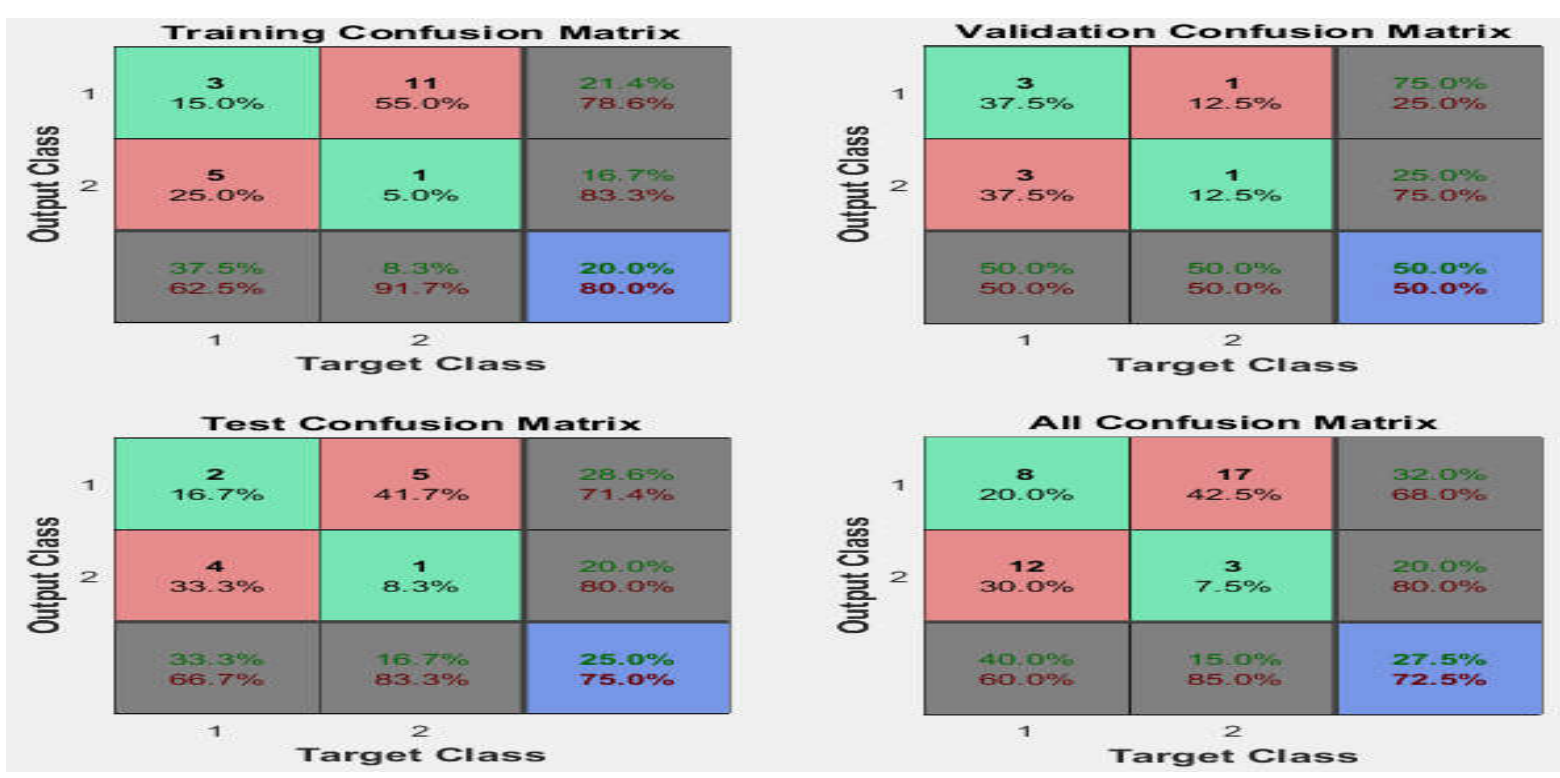

(a)

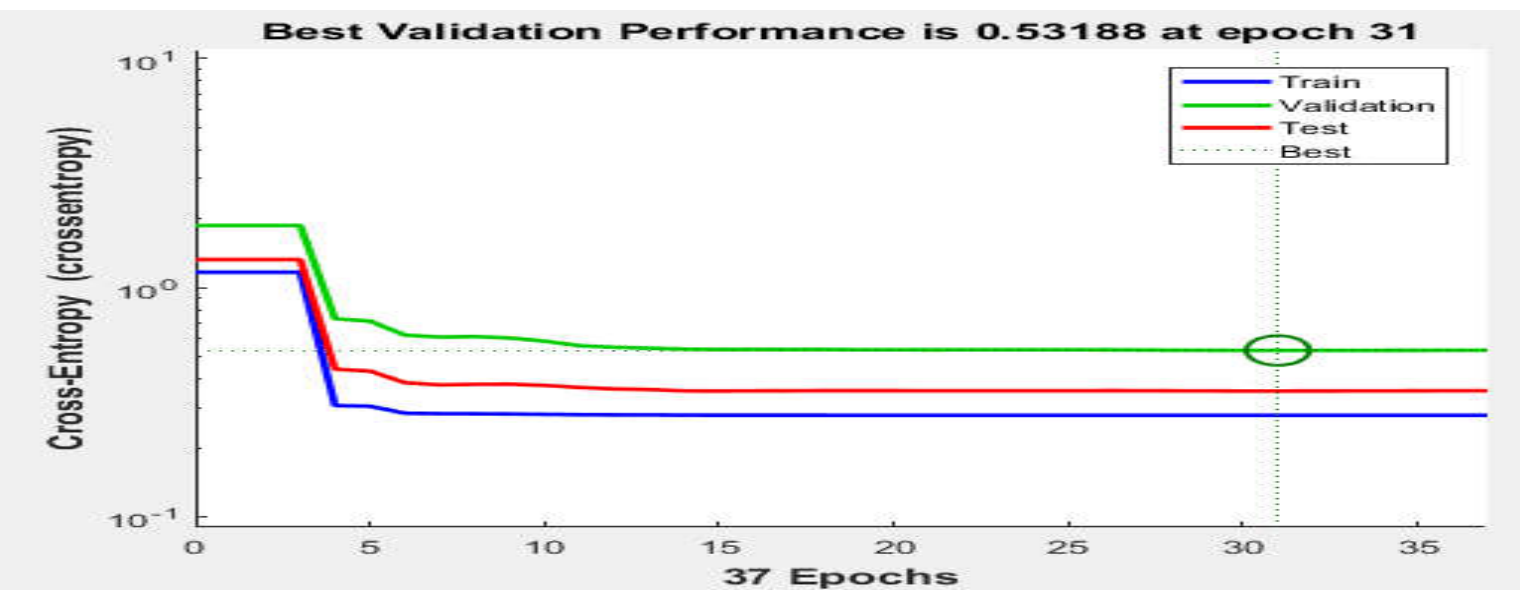

(b)

Fig.5. (a) Confusion Matrix and (b) MLPNN performance for Zero-mean PSD feature 


\section{CONCLUSION}

In summary, for MLPNN classifier, we can conclude that Zero-mean PSD feature produces highest accuracy compared to Baseline PSD featured and other methods such as Softmax, Mag-scale as well as other much more complicated non-linear methods such as LLE and OLS when used in classifying Dataset 1 and Dataset 2 respectively. For SVM, PCA and Zero-mean feature produces the best accuracy at $91.67 \%$ accuracy for Dataset 1 and Dataset 2 respectively. From the results, we found that MLPNN classification could be improved further for since the accuracy achieved in all tested cases is less than 70\% for MLPNN. SVM classifier performs significantly better than MLPNN in classifying PSD features. The best SVM performance for Dataset 1 is $91.67 \%$, while the best MLPNN performance is only $66.00 \%$. In terms of the performance improvements acquired from modifications of PSD features, for MLPNN, Zero-mean normalization is around 5\% better than baseline PSD. While for SVM, PCA features are 10\% better than baseline PSD. Feature extraction methods other than PSD such as Power Spectral Entropy (PSE) and Discrete Wavelet Transform (DWT) may provide better performance, but further studies are needed to prove this. Besides, we identify that number of neurons in hidden layer also play important role in producing the better result in MLPNN. Finally, better technique with less computational time and high efficiency is one of the factors that will influence better result too.

\section{ACKNOWLEDGEMENTS}

The work presented here is sponsored by UniversitiTeknologi MARA Malaysia under grant 600-IRMI/DANA 5/3/SINERGI (0001/2016).

\section{REFERENCES}

[1] Millett D.Hans Berger: From psychic energy to the EEG.Perspectives in Biology And Medicine, 2001, 44(4):522-542

[2] Khalid M B, Rao N I, Rizwan-i-Haque I, Munir S, Tahir F. Towards a brain computer interface using wavelet transform with averaged and time segmented adapted wavelets. In 2nd International Conference on Computer, Control and Communication, 2009, pp. 1-4

[3] Kaufmann T, Herweg A, Kübler A. Toward brain-computer interface based wheelchair control utilizing tactually-evoked event-related potentials. Journal of NeuroEngineering and Rehabilitation, 2014, 11(1):1-17 
[4] Carlson T, Millan J D. Brain-controlled wheelchairs: A robotic architecture.IEEE Robotics and Automation Magazine, 2013, 20(1):65-73

[5] Denuelle A, Thurrowgood S, Strydom R, Kendoul F, Srinivasan M V. Biologically-inspired visual stabilization of a rotorcraft UAV in unknown outdoor environments. In International Conference on Unmanned Aircraft Systems, 2015, pp. 1084-1093

[6] El Diwiny M, El Sayed A H, Hassanen E S, El Magd G A. PTSD monitoring by using brain computer interface for unmanned aerial vehicle operator safety. In IEEE/AIAA 33rd Digital Avionics Systems Conference, 2014, pp. 1-6

[7] Anindya S F, Rachmat H H, Sutjiredjeki E. A prototype of SSVEP-based BCI for home appliances control. In 1st International Conference on Biomedical Engineering, 2016, pp. 1-6 [8] Bian Y, Wang X, Zhao L, Cui S. A new control system for space robot based on brain-computer interfaces. In IEEE Advanced Information Management, Communicates, Electronic and Automation Control Conference, 2016, pp. 232-236

[9] Chuan S, Duanfeng C, Wei Y, Chaozhong W, Fei T.A design of brain-computer interface control platform for intelligent vehicle. In International Conference on Transportation Information and Safety, 2015, pp. 70-74

[10] Bi L, Fan X A, Jie K, Teng T, Ding H, Liu Y. Using a head-up display-based steady-state visually evoked potential brain-Computer interface to control a simulated vehicle. IEEE Transactions on Intelligent Transportation Systems, 2014, 15(3):959-966

[11] Valipour S, Shaligram A D, Kulkarni G R. Detection of an alpha rhythm of EEG signal based on EEGLAB.International Journal of Engineering Research and Applications, 2014, 4(1):154-159

[12] Stoica P., Moses R. L.Spectral analysis of signals. New Jersey: Prentice-Hall, 2005

[13] Schuster A. On lunar and solar periodicities of earthquakes. Proceedings of the Royal Society of London, 1897, 61(369-377):455-465

[14] Güntekin B, Başar E. A new interpretation of P300 responses upon analysis of coherences. Cognitive Neurodynamics, 2010, 4(2):107-118 
[15] Al-Ayyoub M, Al-Rahman A H, Qadoumi Y, Hani M B, Masadeh M, Al-Asal R A, Aboud M B. Mind-controlling green unmanned vehicles through the cloud: Building a prototype. Journal of Advances in Information Technology, 2015, 6(1):34-38

[16] Gomez-Gil J, San-Jose-Gonzalez I, Nicolas-Alonso L F, Alonso-Garcia S. Steering a tractor by means of an EMG-based human-machine interface. Sensors, 2011, 11(7):7110-7126

[17] Chen S, Cowan C F, Grant P M. Orthogonal least squares learning algorithm for radial basis function networks. IEEE Transactions on Neural Networks, 1991, 2(2):302-309

[18] Roweis S T, Saul L K. Nonlinear dimensionality reduction by locally linear embedding. Science, 2000, 290(5500):2323-2326

[19] Chang C C, Lin C J. LIBSVM: A library for support vector machines. ACM Transactions on Intelligent Systems and Technology, 2011, 2(3):1-27

[20] Millan J R. On the need for on-line learning in brain-computer interfaces. In IEEE International Joint Conference on Neural Networks, 2004, pp. 2877-2882

[21] Fadhlan H K Z, Md. Hazrat A, Amir A S, Zairi I R.Development of mobile face verification based on locally normalized gabor wavelets. International Journal on Advanced Science, Engineering and Information Technology, 2017, 7(3):1026-1031

\section{How to cite this article:}

Zaman F H K, Shukur N A M, Hamzah, Zaini N M, Rizman Z I. Performance of modified power spectral density features in eeg signal classification. J. Fundam. Appl. Sci., 2017, 9(3S), $830-843$ 